\title{
Il sistema politico partitico durante la Prima repubblica portoghese (1910-1926)
}

Manuel Baiôa

\section{I \\ Il Partido republicano português: il partito predominante della Prima repubblica portoghese}

Durante la Prima repubblica portoghese (1910-1926) il Partido republicano português (Prp), che aveva capeggiato la rivoluzione repubblicana del ı9ıo, riuscì a diventare il partito predominante - quasi egemonico - del sistema pluripartitico ${ }^{\mathrm{I}}$ e a accorpare in sé molti interessi, il che contribuì a farlo divenire un partito poco omogeneo ${ }^{2}$.

Il predominio del Prp e l'assenza di un'alternanza provocarono un'identificazione tra il partito e il regime dovuta al suo controllo sull'apparato statale e alla difficoltà delle forze di opposizione ad accedere in maniera indipendente agli organi di potere. Questa situazione provocò una crisi di rappresentanza e di partecipazione politica e rafforzò la delegittimazione e l'instabilità della repubblica $^{3}$. Fu a partire da questo periodo che iniziarono a sorgere, tramite le varie dissidenze, tutti gli altri partiti rilevanti della Prima repubblica con un origine diversa da quello repubblicano: a destra il Partido monárquico, il Centro católico português e l'União dos Interesses Económicos; a sinistra il Partido socialista e il Partido comunista português. La nascita dei nuovi partiti contraddistinse il regime repubblicano anche grazie al forte desiderio dei dirigenti del Prp di mettersi a capo di un movimento frontista nel governo e nel parlamento che riunisse tutti i componenti della "famiglia" repubblicana spaccata.

Il dominio del sistema politico da parte del Prp è stato spiegato con motivazioni storiche, organizzative e costituzionali, e sottolineando il ricorso fatto a pratiche

I. M. Rebelo de Sousa, Os Partidos Políticos no Direito Constitucional Português, Braga, Livraria Cruz, 1983, p. 167; F. Farelo Lopes, Um regime parlamentarista de partido dominante, in Portugal Contemporâneo, a cura di A. Reis, vol. III, Lisboa, Publicações Alfa, I990, pp. 85-I00.

2. A.J. Sousa Monteiro de Queirós, A Esquerda Democrática e o final da Primeira República, Lisboa, Livros Horizonte, 2008.

3. M. Rebelo de Sousa, Os Partidos Políticos no Direito Constitucional Português, cit., pp. I67-I77; F. Farelo Lopes, Poder Político e Caciquismo na r. ${ }^{a}$ República Portuguesa, Lisboa, Editorial Estampa, I994, p. II; E. Castro Leal, Parties and Political Identity: the Construction of the Party System of the Portuguese Republic (I9I0-1926) in «e-Journal of Portuguese History», vol. 7, n. I, 2009, pp. 37-44. 
violente e clientelari. Il fatto che il Prp già durante la monarchia avesse fatto propaganda diffondendo l'ideologia repubblicana, e fosse stato il protagonista della rivoluzione del 5 ottobre ı9ıо, lasciò segni profondi nel popolo e nelle élite repubblicane. Questa base sociale di appoggio iniziale, con l'aggiunta di alcuni dirigenti dei vecchi partiti monarchici, gli permise di creare una fitta rete organizzativa e una forte influenza in tutto il Paese, specie se comparata con la debole struttura dei suoi due principali rivali, il Partido republicano evolucionista (I9I2-I9I9) e l'União republicana (1912-1919). I leader politici di questi due partiti moderati, António José de Almeida e Brito Camacho, insieme ad altri esponenti, lasciarono il Prp alla fine del I9II, dovendo così rinunciare anche al nome ufficiale del partito che era riuscito a proclamare la repubblica in Portogallo e alla forte macchina organizzatrice che comprendeva commissioni politiche, giornali e centri associativi-politici in quasi tutte le città e le cittadine portoghesi. Il Prp aveva così la strada spianata per conquistare progressivamente le risorse dello Stato: Afonso Costa presiedette il primo governo monopartitico della repubblica a partire dal 9 gennaio ı19; nelle elezioni supplementari di novembre del i9ı3 il Prp ottenne la maggioranza assoluta del Parlamento; nel gennaio I9I4 si insediarono le prime giunte comunali uscite dalle elezioni che rafforzarono il dominio del Prp in provincia. Il controllo del potere centrale e di quello locale rafforzarono il caciquismo esercitato dal Prp 4 , anche grazie alle risorse che si resero disponibili per i suoi sostenitori.

Anche la legge elettorale fu usata in funzione degli interessi del Prp. I repubblicani, che avevano sempre difeso l'adozione del suffragio universale maschile durante la monarchia, nel I9I approvarono una legge che estese il diritto di voto a tutti i cittadini con più di 2I anni che sapessero leggere e scrivere o che fossero capi famiglia. In questo modo il $\mathrm{I} 4,3 \%$ della popolazione portoghese riuscì ad avere accesso al voto compiendo un passo avanti verso il suffragio universale maschile.

Tuttavia, vista la formazione dei nuovi partiti repubblicani, e il pericolo che i partiti monarchici partecipassero alle elezioni, il Prp fece approvare una nuova legge elettorale nel I9I3 che limitava la facoltà di voto ai soli cittadini maschi che sapessero leggere e scrivere, per questo appena il $7,7 \%$ della popolazione poté partecipare alle elezioni di quell'anno, dato che l'analfabetismo colpiva il $69 \%$ della popolazione portoghese. Questa legge rafforzò il tipo di clientelismo che il Prp poteva offrire - l'accesso ai servizi e ai ruoli all'interno dell'apparato statale; allontanò dal suffragio le masse analfabete contadine, facilmente convincibili dai notai cattolici, monarchici e conservatori ${ }^{5}$; diluì il clientelismo tradizionale tipico delle società fortemente rurali e rafforzò un clientelismo di transizione, più urbano e amministrativo. Il cacique proprietario terriero si trovò a dover competere con nuovi protettori e intermediari (commercianti, medici, avvocati e funzionari

4. Il Caciquismo è un sistema politico tipico del XIX secolo nel quale un notabile politico locale (il caicique) basa il suo successo elettorale e il suo potere politico attraverso lo scambio di favori con i suoi elettori (i clientes). [n.d.t.]

5. Portugal da Monarquia para a República. Nova História de Portugal, vol. II, a cura di A. H. de Oliveira Marques, Lisboa, Editorial Presença, 199ı, p. 417. 
pubblici) più legati al partito di governo, che controllavano e parcellizzavano certe risorse, beni e servizi. Il clientelismo tradizionale perse così importanza rispetto al clientelismo statale, amministrativo, locale e professionale che era nelle mani del $\operatorname{Prp}^{6}$. Il sistema di suffragio ristretto ai soli uomini alfabetizzati si mantenne per tutta la Prima repubblica, ad eccezione del igi8 durante la presidenza di Sidónio Pais, quando per la prima volta, anche se in forma effimera, si introdusse il suffragio universale maschile, allargandolo a tutti gli uomini sopra ai 2I anni .

Il dominio storico, organizzativo e clientelare del Prp fu anche rafforzato dalle violenze esercitate dai membri del partito contro i loro avversari. La tradizione rivoluzionaria, giacobina e violenta che il partito aveva usato nel periodo monarchico si prolungò nel corso del regime repubblicano, questa volta non solo contro i monarchici, ma anche contro i suoi antichi compagni repubblicani. Aggredire un leader del Partido republicano evolucionista, incendiare o distruggere la sede di un partito o di un giornale monarchico o cattolico, divenne una pratica corrente che la polizia e l'autorità giudiziaria lasciavano correre impunemente ${ }^{8}$.

Infine, il regime costituzionale creato dalla Prima repubblica contribuì a facilitare la predominanza del Prp. La costituzione del ı̀ı creò un sistema politico nel quale la supremazia parlamentare era chiara. Il parlamento, diviso in camera dei deputati e senato, era eletto a suffragio diretto; aveva competenze legislative e fiscali sull'operato del governo e dell'amministrazione pubblica; la facoltà di eleggere il presidente della Repubblica con un mandato di quattro anni, non prorogabile. Il presidente aveva il compito di formare il governo e promulgare le leggi anche se non poteva porre il veto assoluto o sospensivo e non poteva sciogliere il parlamento o prorogarne il funzionamento; inoltre poteva essere destituito con il voto favorevole dei due terzi dei membri del parlamento, cosa che indeboliva il suo mandato. Con la riforma costituzionale del igrs al presidente della Repubblica fu concesso il potere di sciogliere il parlamento, ma solo dopo la preventiva consultazione del Conselho Parlamentar?.

6. F. Farelo Lopes, Poder Político e Caciquismo na I. ${ }^{a}$ República Portuguesa, cit.; J. M. Gonçalves da Silva, O Clientelismo partidário durante a I República: o caso do Partido Reconstituinte (1920-1923), in «Análise Social», vol. XXXII, n. I40, 1997, pp. 3I-74.

7. Legislação eleitoral portuguesa: 1820-1926, a cura di P. Tavares de Almeida, Lisboa, Presidência do Conselho de Ministros, Imprensa Nacional - Casa da Moeda, 1998.

8. Cfr. V. Pulido Valente, O Poder e o Povo. A Revolução de rgro, 2a edizione, Lisboa, Moraes Editores, I982; Id., A República Velha (I910-1917), Ensaio, Lisboa, Gradiva, 1997; R. Ramos, A Segunda Fundação (I890-1926), in História de Portugal, a cura di J. Matoso, vol. VI, Lisboa, Editorial Estampa, 2001.

9. Il Conselho Parlamentar fu creato con la Legge 89i del 22 settembre 1919. Era un organo consultivo del Presidente della Repubblia formato da I8 membri eletti dal parlamento in rappresentanza di tutti gli schieramenti partitici [n.d.t.]. J. Miranda, Manual de Direito Constitucional, 3. ${ }^{\text {a }}$ ed., vol. I, Coimbra, Coimbra Editora, 1985, pp. 260-298; L. Salgado de Matos, Tudo o que sempre quis saber sobre a Primeira República em 37 mil palavras, Imprensa de Ciências Sociais, Lisboa, 2oro; A. de Araújo; L. Bigotte Chorão, Politica e Direito nos Alvores da Primeira República, in Outubro: A Revolução Republicana em Portugal (IgI0-I926), a cura di Luciano Amaral, Edições 70, Lisboa, 20II, pp. II7-I40; A Assembleia Constituinte e a Constituição de IgII, Lisboa, Assembleia da República, 2oII. 
In conclusione, il Prp, dopo essere stato a capo della rivoluzione del 5 ottobre I9IO, e aver conseguito una chiara maggioranza nel Parlamento nel I9II e, soprattutto nel 1913, rafforzò la sua egemonia nel sistema politico, tanto che, in futuro, le forze di opposizioni avrebbero avuto molte difficoltà ad accedere all'apparato governativo per via costituzionale.

2

\section{L'opposizione nei confronti del Partido Republicano Português}

L'incontestato dominio del Prp si tramutò in un'autentica "dittatura" per l'opposizione, che sentiva che difficilmente avrebbe potuto ottenere l'alternanza attraverso le elezioni senza ricorrere a pratiche anticostituzionali o violente.

L'inizio della prima guerra mondiale e la decisione del Prp di partecipare a fianco degli alleati rafforzò l'intervento dei militari nella politica e aumentò le tensioni nella società e nei partiti portoghesi. Il presidente della Repubblica, Manuel de Arriaga, vicino alle posizioni dei repubblicani conservatori, varò il 25 gennaio I9I5 un governo di nomina presidenziale, con a capo il generale Pimenta de Castro e costituito prevalentemente da militari, senza l'appoggio del parlamento. Il Prp considerò questa decisione anticostituzionale, come se si trattasse di una "dittatura", e pertanto, il I4 maggio dello stesso anno, reagì in maniera violenta. Il ritorno del Prp al potere non scoraggiò l'opposizione a proseguire con la sua strategia tanto che Machado Santos, l'eroe dell'instaurazione della Repubblica, tentò un colpo di Stato il I3 dicembre 19ı6, e Sidónio Pais fece lo stesso il 5 dicembre i917. Se il primo ne uscì indebolito, il secondo dette il via ad un regime repubblicano alternativo che, in un primo momento, riuscì a raggruppare tutte le fazioni contrarie alla politica intrapresa fin dal ıэı dal Prp.

Il regime capeggiato da Sidónio Pais, conosciuto come "Sidonismo" o "Nuova Repubblica" (dicembre 1917-dicembre I9I8), anticipò alcune soluzioni politiche che sarebbero state intraprese dalle dittature europee autoritarie e fasciste negli anni venti e trenta. All'interno di un sistema politico presidenzialista e populista, con Sidónio Pais ci fu un recupero dei valori tradizionali, in particolare quello della patria, e una valorizzazione della leadership carismatica e dell'ordine. Lo Stato intervenne attivamente contro la plutocrazia e represse il movimento operaio e repubblicano di sinistra. Dall'altra parte Sidónio Pais cercò di andare verso una nuova organizzazione politica ed oltrepassare le divisioni create dal liberalismo e dal repubblicanesimo, avvicinandosi ai cattolici, ai monarchici e ad altri attori sociali banditi dagli organi del potere dal ıюro. Cercò di scavalcare i partiti politici e il parlamento, intesi come forme di rappresentazione degli interessi nazionali, grazie alla creazione di organismi corporativi, alla formazione di un primo embrione di partito unico che aggregasse le frange conservatrici (Partido nacional republicano) e al nuovo ruolo di mobilizzazione del "capo"

Io. A. J. Telo, Sidónio Pais - A Chegada do Século XX. A Primeira República Portuguesa. Entre o Liberalismo e o Autoritarismo, a cura di N. Severiano Teixeira, A. Costa Pinto, Lisboa, Edições Colibri, 
La corrente di opinione che maggiormente difendeva il presidenzialismo autoritario contrario al parlamentarismo salì al governo solo con Sidónio Pais, ma aveva già dato mostra della sua presenza ai tempi della propaganda repubblicana durante il periodo monarchico. Il forte impatto provocato dalla partecipazione del Portogallo nella Grande guerra permise un'alleanza tattica tra i vari settori contrari alla politica del Prp. Inizialmente Sidónio Pais ebbe l'appoggio del suo partito (União republicana), del Partido centrista republicano (una dissidenza del Partido republicano evolucionista), e di settori apparentemente divergenti: monarchici, cattolici e operai ${ }^{\text {II }}$. Questo blocco si sfaldò quando Sidónio Pais decise di creare un regime presidenzialista. La fondazione, nell'inizio di aprile, di un partito di appoggio al regime (Partido nacional republicano), nel quale si fuse il Partido Centrista Republicano, e l'elezione simultanea del presidente della Repubblica e del Parlamento il 28 aprile i9ı8, provocarono le dimissioni dal governo dei tre membri dell'União republicana e l'inizio di una politica di distanziamento dal regime. Sidónio Pais fu eletto presidente della Repubblica senza opposizione in un sistema di suffragio maschile universale; il partito di regime ottenne la maggioranza assoluta nella camera dei deputati (I08 deputati su 155) grazie all'aiuto della censura e alla mancata partecipazione dei principali partiti repubblicani. I monarchici, che parteciparono per la prima volta alle elezioni dopo la proclamazione della Prima repubblica, elessero 37 deputati, i cattolici e gli indipendenti 5 . Continuò ad esistere un partito dominante nel parlamento, questa volta di carattere conservatore, ma le tensioni e le divergenze a sinistra e a destra si intensificarono, specialmente quando il sidonismo non adempì a tutte le sue rivendicazioni. Il parlamento e il Partido nacional republicano ebbero un'azione irrilevante e poco mobilizzatrice all'interno del regime e restarono sottomessi all'azione del presidente, il che risollevò le divergenze interne $\mathrm{I}^{\mathrm{I} 2}$.

Il vuoto di potere venutosi a creare con l'assassinio di Sidónio Pais il I4 dicembre I9r8 portò il Paese ad una guerra civile. Nel nord del Portogallo, il ı9 gennaio I919, fu proclamata una monarchia e quattro giorni dopo scoppiò un'insurrezione monarchica a Lisbona. Il pericolo monarchico distrusse ciò che restava del blocco "sidonista" e unì temporaneamente i repubblicani in disaccordo in difesa delle istituzioni. In poche settimane la rivolta monarchica fu soffocata.

Dopo il sidonismo sembrava che si fosse tornati alla situazione politica della prima fase della Repubblica. L'élite sidonista, raggruppata intorno al Partido nacionalista republicano, s'indebolì e si divise. Un gruppo formò il Partido repu-

200o, pp. II-24; F. Ribeiro de Meneses, Sidónio Pais, the Portuguese "New Republic" and the challenge to liberalism in southern Europe, in «European History Quarterly», vol. 28, n. I, 1998, pp. I09-130; A. B. Malheiro da Silva, Sidónio e Sidonismo, Coimbra, Imprensa da Universidade de Coimbra, 2006.

II. Cfr. A. J. Telo, O Sidonismo e o Movimento Operário Português, Lisboa, I977; M. Braga da Cruz, As origens da Democracia Cristã em Portugal e o Salazarismo, Lisboa, Ed. Presença, i98o; M. A. Samara, Verdes e Vermelhos. Portugal e a Guerra no ano de Sidónio Pais, Lisboa, Editorial Notícias, 2002; M. Dias dos Santos, Os Monárquicos e a República Nova, Coimbra, Quarteto, 2003.

I2. F. Ribeiro de Meneses, Sidónio Pais e o Sidonismo in, Outubro: A Revolução Republicana em Portugal (I9I0-I926), a cura di L. Amaral, Lisboa, Edições 70, 20II, pp. II7-I40, pp. 227-256. 
blicano conservador con l'obiettivo di creare un sistema bipartitico di alternanza, che però si sarebbe sciolto nel 1920. Un altro gruppo fondò il Partido nacional presidencialista con l'obiettivo di portare avanti l'eredità del sidonismo, rilanciando il corporativismo e la dimensione antiliberale; tuttavia, ebbe un'importanza marginale nel sistema dei partiti della Prima repubblica, benché i suoi membri fossero presenti in un variegato ventaglio di organizzazioni conservatrici. Una parte della sua élite entrò nel Partido republicano liberal (Prl) nel ı9ı9; un'altra parte avrebbe integrato nel 1925 il settore conservatore repubblicano, rappresentato dal Partito republicano nacionalista; infine, un'ultima parte partecipò al processo di fondazione dei gruppi radicali nazionalisti prossimi al fascismo. Non tenendo conto della situazione venutasi a creare Machado Santos continuò a non volersi integrare nei partiti tradizionali, e fondò, nel ı19, la Federação nacional republicana. All'interno dei partiti repubblicani tradizionalisti sembrava che niente fosse cambiato. Il Prp ottenne la maggioranza assoluta nel parlamento nelle elezioni del in maggio del I9ı e vide eletti 86 dei 163 deputati che componevano la camera. I partiti repubblicani conservatori diminuirono nella maggior parte delle circoscrizioni elettorali, il che contribuì a far loro ottenere dei risultati modesti, anche se superiori in relazione alle elezioni del i9is. Il Partido republicano evolucionista elesse 38 deputati e l'União republicana $\mathrm{I} 7$, il che li rendeva le principali forze di opposizione, ma senza la possibilità di rappresentare un'alternativa all'interno di un parlamento saldamente controllato dal Prp. Gli indipendenti e i socialisti videro crescere significativamente la loro influenza in queste elezioni del dopoguerra, ottenendo i primi 13 deputati e i secondi 8 . I cattolici riuscirono nuovamente ad eleggere un deputato, ma ridussero la loro rappresentanza parlamentare rispetto al Sidonismo e i monarchici tornarono a non avere le condizioni politiche per presentarsi alle urne, vista la recente insurrezione monarchica e la conseguente violenza e coercizione repubblicana ${ }^{13}$.

Nel frattempo, la situazione politica si presentava profondamente alterata dopo la partecipazione del Portogallo nella prima guerra mondiale e le esperienze "autoritarie" di Pimenta de Castro e di Sidónio Pais. I partiti repubblicani e i politici erano profondamente divisi fra interventisti e neutralisti; fra i sostenitori di Pimenta de Castro ed i fautori della rivoluzione del I4 maggio igrs e tra i sidonisti e i "veri repubblicani”. Questi risentimenti, aggravati dagli odi personali, riguardarono internamente tutti i partiti. Afonso Costa, il leader carismatico del Prp durante la Prima repubblica (I9IO-I9I7), si allontanò dal Paese e dal suo partito profondamente ferito dalle incomprensioni di cui era stato vittima. La condizione che pose per un suo eventuale ritorno al governo era impossibile: l'unione di tutti i partiti repubblicani. Neanche dentro lo stesso Prp questo fu possibile; era infatti difficile trovare una strategia e un leader comune nel partito dopo il ı9ı. L'organico interno dei partiti repubblicani assenti nelle direzioni e contrari al presidenzialismo contribuì

13. E. Castro Leal, António Ferro. Espaço Político e Imaginário Social (I9I8-32), Lisboa, Edições Cosmos, 1994, pp. 97-I53; Id., Partidos e Programas. O campo partidário republicano português (I9Io1926), Coimbra, Imprensa da Universidade de Coimbra, 2008, pp. 59-II8. 
ulteriormente ad accentuare i conflitti interni e le scissioni. I nuovi candidati a leader del Prp, António Maria da Silva, Domingos Pereira, e Àlvaro de Castro, misero in discussione le rispettive strategie e i loro uomini all'interno del partito e nel parlamento, arrivando al punto che i governi espressione del Prp caddero per i voti contrari espressi proprio dai deputati dello stesso partito, come nel caso del governo di Sá Cardoso il 2r gennaio I920, e di Domingos Pereiras il 4 marzo I920. Tra il marzo e l'aprile 1920 Àlvaro de Castro, insieme a is deputati e io senatori abbandonò il Prp. Nei mesi seguenti, altri parlamentari si sarebbero uniti al suo gruppo formando il Partido republicano de reconstituição nacional che arrivò a contare 33 deputati e Io senatori ${ }^{\mathrm{I}}$. Nel novembre 1920 anche Domingos Pereira, insieme ad altri militanti, dette vita ad una nuova dissidenza all'interno del Prp, che si sarebbe successivamente reintegrata nel partito nel dicembre I92I. Il Prp smise di avere la maggioranza assoluta alla Camera nel marzo ig2o e la riottenne solo nel dicembre 1925. Questa situazione fu causata dall'assenza sistematica di alcuni deputati durante le sessioni parlamentari, ma principalmente, dalle scissioni e dalle slealtà all'interno del partito.

La mancanza di unità non riguardava solo il Prp; anche il Partido republicano evolucionista, l'União republicana e il Partido monárchico attraversarono periodi di instabilità interna. Gli scarsi risultati elettorali ottenuti dai due partiti repubblicani conservatori nel maggio I9I9 portò a riconoscere l'errore commesso nel mese passato di rifiutare la fusione delle destre repubblicane in un partito alternativo al Prp. Questa fusione si concretizzò nell'ottobre 1919, con la nascita del Partito republicano liberal. I leader storici dei due antichi partiti repubblicani, António José de Almeida e Brito Camacho, si stavano ritirando progressivamente dalla politica dei partiti; il primo perché eletto presidente della Repubblica nell'agosto I9ı9, e il secondo perché ricopriva l'incarico di alto commissario per il Mozambico tra il I920 e il I923. Il nuovo presidente, António José de Almeida, vide il suo potere rinforzato grazie alla revisione costituzionale del I9ı che dava al capo dello Stato la possibilità di dissolvere il parlamento, dopo il parere del Conselho Parlamentar. Questo cambiamento costituzionale dava al partito che dominava l'esecutivo l'opportunità di manovrare le elezioni in suo favore il che, prevedibilmente, avrebbe spezzato l'invincibilità del Prp. Intanto, anche il Prl stava attraversando un periodo di difficoltà, diviso internamente a causa dei persistenti legami dei suoi membri con le vecchie affiliazioni partitiche e dal passaggio di alcuni parlamentari, insoddisfatti della fusione, verso il Partido Popular. Quest'ultimo aveva adottato nel frattempo un orientamento di sinistra riuscendo ad attrarre tra le sue fila politici di diversi partiti, in particolare di antichi membri del $\operatorname{Prp}^{\mathrm{IS}}$.

I4. J. M. Garcia Salazar Gonçalves da Silva, O Partido Reconstituinte: Clientelismo, faccionalismo e a descredibilização dos partidos políticos durante a Primeira República (I920-1923), Tesi di maestrado, Lisboa, Instituto de Ciências Sociais da Universidade de Lisboa, I996, pp. 36-39.

I5. A.H. de Oliveira Marques, $A I^{a}{ }^{a}$ República Portuguesa (alguns aspectos estruturais), Lisboa, Livros Horizonte, I980, pp. I26-I28; J. M. Garcia Salazar Gonçalves da Silva, O Partido Reconstituinte, cit., pp. 38-39 e 300-30I; L. Farinha, Estudo sobre a acção política parlamentar de Francisco Pinto Cunba 
Questa frammentazione alterò leggermente il sistema dei partiti. Dal ı9ıo al I9I7 esisteva infatti un multipartitismo circoscritto ad un partito dominante; mentre dal ı9ı9 al 1926 si passò ad avere un multipartitismo frammentato con un partito dominante, caratterizzato da una polverizzazione crescente delle forze politiche. Il Prp continuò ad avere un ruolo predominante, anche se più fragile ${ }^{16}$.

La violenza politica e sociale aumentò all'inizio degli anni venti a causa del deterioramento delle condizioni di vita nel contesto del dopoguerra. L'incapacità dei governi di risolvere i problemi finanziari e di ordine pubblico, associata alla maggiore visibilità dei militari dopo la Prima guerra mondiale, assoggettò il potere civile a quello militare. I membri delle forze armate passarono a ricoprire un ruolo più rilevante nei partiti, nel Parlamento e nei governi; un fatto unico nell'Europa del tempo. A somiglianza di quanto avvenne in Spagna, anche in Portogallo si formarono delle "giunte militari" nel I9I8 e nel ı9ı9; tuttavia, la maggiore partecipazione dei militari nelle istituzioni politiche della Prima repubblica non permise di risolvere i problemi corporativi e finanziari che riguardavano un gran numero di ufficiali portoghesi nel dopoguerra. Una parte dei militari smise di identificarsi con il potere politico e con il Prp e fu protagonista di innumerevoli rivolte ${ }^{17}$.

La frammentazione e l'indisciplina partitica associata al problema dell'ordine pubblico e all'interferenza dei militari nelle istituzioni politiche provocarono una instabilità governativa crescente. Il presidente della Repubblica, tra il Is gennaio del I920 e il 2 marzo I92I, nominò dieci governi con varie combinazioni partitiche. Il parlamento eletto nel ıوı era cambiato molto dai precedenti a causa delle dissidenze, degli accorpamenti e del disordine interno delle forze politiche. I piccoli partiti come il Partido republicano de reconstituição nacional e il Partido popular passarono a ricoprire un ruolo fondamentale nella formazione degli esecutivi grazie alle intese parlamentari con quelli maggiori (Prp e Prl). Era evidente che non era più possibile trovare una soluzione governativa stabile con quel tipo di parlamento. Questa era l'opportunità che da anni aspettavano i repubblicani conservatori. Il presidente della Repubblica, António José de Almeida, grazie alla revisione costituzionale, poté nominare un governo espressione del Prl e sciogliere il parlamento. Le nuove elezioni, che si tennero il Io luglio I92I, dettero, come sempre, la vittoria al partito che era al governo. Il Prl vide eletti 79 deputati, il Prp 54 e il Partido repulicano de reconstituição nacional $\mathrm{I} 2$. I restanti I8 seggi della camera furono distribuiti tra le formazioni minori che poterono così ricoprire un ruolo importante data la maggioranza chiara, ma non assoluta, che il Prl aveva conseguito. I monarchici, che finalmente avevano riacquistato le condizioni necessarie per presentarsi alle urne,

Leal como Deputado ao Congresso da República (I918-1926), Porto, Edições Afrontamento/Assembleia da República, 2002.

I6. M. Rebelo de Sousa, Os Partidos Políticos no Direito Constitucional Português, cit., p. I73.

17. Cfr. Maria Carrilho, Forças Armadas e Mudança Política em Portugal no século XX. Para uma explicação sociológica do papel dos militares, Lisboa, Imprensa Nacional - Casa da Moeda, I985; José Medeiros Ferreira, O comportamento político dos militares. Forças armadas e regimes políticos em Portugal, Lisboa, Ed. Estampa, 1992. 
ottennero 4 deputati, gli indipendenti 5 , i cattolici 3 , i dissidenti 3 , i regionalisti 2 e i popolari $\mathrm{I}^{\mathrm{I}}$.

La durata del nuovo governo, espressione del Prl presieduto da António Granjo, tuttavia, sarebbe stata breve e sarebbe finita tragicamente. Le conseguenze sociali, economiche e sociali del dopoguerra si accentuarono nell'estate del r92r e il governo si trovò costretto a prendere alcuni provvedimenti impopolari che portarono ad un'insurrezione di sinistra sfociata nel golpe del i9 ottobre i92I. Il governo, verificata l'impossibilità di resistere, si dimise; nonostante ciò esponenti di una corrente radicale sequestrarono e uccisero l'ex presidente António Granjo, e altre importanti figure del regime repubblicano. Questo accadimento, conosciuto come noite sangrenta, segnò profondamente le élite e l'opinione pubblica portoghese: fu dimostrata la fragilità delle istituzioni repubblicane e fu provato che la repubblica era solo apparentemente un regime democratico, dato che non permetteva un'alternanza dei partiti al governo, cosa abituale nei regimi elitisti del XIX secolo.

La noite sangrenta accentuò il discredito e la delegittimazione dei partiti politici, della classe dirigente portoghese e della stessa repubblica. Tuttavia fu uno stimolo affinché i politici repubblicani prendessero coscienza che era necessario indire una tregua per poter finalmente governare con una certa stabilità. Un patto stabilito tra i principali partiti repubblicani permise la formazione di un governo di coalizione comprendente i membri del Prp, del Prl, del Partido repulicano de reconstituição nacional e gli indipendenti, guidato da Cunha Leal, che indisse nuove elezioni legislative per il 22 gennaio 1922. Il Prp vinse nuovamente le elezioni, ma senza ottenere la maggioranza assoluta (7I deputati su 163). Il Prl fu la seconda forza più votata con 33 deputati, seguita dal Partido repulicano de reconstituição nacional con 17 e dal gruppo di Cunha Leal con I3. I partiti repubblicani minoritari trassero vantaggio dalla perdita di prestigio del Prp e dalla divisione interna del Prl, nel caso di una costituzione di un governo di coalizione tra repubblicani. Tuttavia, il Prp, grazie all'avvicinamento di 5 deputati cattolici, di 2 regionalisti e all'appoggio di 5 indipendenti, che lo stesso Prp aveva contribuito a far eleggere, riuscì ad ottenere una maggioranza parlamentare sufficiente per formare un governo presieduto da António Maria da Silva ${ }^{19}$.

La nuova tendenza conciliatrice e conservatrice del Prp e il riavvicinamento con la Chiesa dopo la noite sangrenta spinse alcuni radicali di sinistra ad uscire dal Prp, ad allearsi con alcuni membri del Partido popular e a dare origine al Partido republicano radical. Questa scissione non eliminò del tutto la frangia di sinistra del Prp che continuò ad avere divergenze interne, ideologiche e personali tra gli ordeiros (la parta conservatrice capeggiata da António Maria da Silva) e i canhotos (la linea i sinistra con a capo José Domingues dos Santos). Questo conflitto latente divenne palese nel luglio del 1925 quando un gruppo di parlamentari canhotos si unì

I8. Cfr. A.H. de Oliveira Marques, $A$ I. ${ }^{a}$ República Portuguesa, cit., pp. I26-I28.

19. Cfr. J.M. Garcia Salazar Gonçalves da Silva, O Partido Reconstituinte, cit., pp. 300-30I; L. Farinha, Cunba Leal, Deputado e Ministro da República. Um notável rebelde, Lisboa, Texto Editores, 2009, pp. 67-I39. 
all'opposizione per destituire il governo presieduto da António Maria da Silva. I parlamentari canhotos furono espulsi dal partito e formarono il Partido republicano de esquerda democratica. Il Prp, pur continuando a vincere le elezioni, divenne sempre più isolato e accerchiato. I conservatori continuavano a non aver fiducia nel Prp a causa della sua eredità giacobina e i radicali se ne erano allontanati a causa del suo imborghesimento e della crescente sintonia con i partiti della destra ${ }^{20}$.

Anche tra i conservatori le divergenze continuavano a permanere; tuttavia, la prospettiva di un conflitto interno al $\operatorname{Prp}$ fu un incentivo per un maggiore sforzo per mantenere l'unità. Dopo alcuni mesi di negoziazioni si formò, nel febbraio I923, il Partido republicano nacionalista che riuniva il Partido republicano de reconstituição nacional e il Prl nel quale erano confluiti da poco Cunha Leal e i suoi protetti. Questo nuovo partito repubblicano conservatore nasceva con l'aspirazione di diventare l'alternativa di destra del Prp all'interno di una repubblica che avrebbe dovuto accogliere tutti i portoghesi. Rapidamente cominciò un'azione ostruzionista nel parlamento contro il governo democratico di António Maria da Silva, che portò all'astensione dai lavori parlamentari tra il 2 maggio e il 22 giugno del 1923 e tra il i8 febbraio e il 22 aprile del 1925 . I membri del Partido republicano nacionalista tentarono anche di influenzare il presidente della Repubblica in modo da costringerlo a sciogliere le camere e a nominarli a capo di un nuovo governo, potendo cosí manovrare a suo piacimento le elezioni. Il presidente della Republica Manuel Teixeira Gomes, invece, decise di provare a formare un "governo nazionale", presieduto da Afonso Costa, che avrebbe dovuto vedere la partecipazione e la collaborazione del Partido republicano nacionalista. I repubblicani nazionalisti, tuttavia, si rifiutarono di formare un governo con il Prp, e quindi Afonso Costa si esiliò nuovamente a Parigi. Le divergenze interne al Prp fecero sì che il is novembre I923 venisse nominato un esecutivo di minoranza presieduto da António Ginestal Machado con il Partido republicano nacionalista. Questo governo, tuttavia, ebbe un carattere effimero a causa della rivolta militare del so dicembre dello stesso anno, del rifiuto del presidente della Repubblica a sciogliere le camere e dello scarso sostegno nel parlamento. La caduta del governo del Partido republicano nacionalista aggravò le divergenze tra alcuni dei suoi membri, e un gruppo capeggiato da Àlvaro de Castro si scisse formando il Grupo parlamentar de acção republicana. Àlvaro de Castro, il I8 dicembre 1923, fu chiamato a presiedere il successivo governo che godeva dell'appoggio minoritario del Prp. Il Partido republicano nacionalista fu minato da una nuova scissione interna nel marzo 1926 quando Cunha Leal e suoi seguaci abbandonarono il IV congresso del partito per formare l'União liberal republicana ${ }^{21}$.

20. Cfr. R. Ramos, A Segunda Fundação (I890-1926), cit., pp. 535-572; A.J. Sousa Monteiro de Queirós, A Esquerda Democrática e o final da Primeira República, cit.; F. Rosas, Porque venceu e porque se perdeu a I República, Lisboa, Bertrand Editora, 20Io, pp. 65-I25.

21. Cfr. R. Leite Pinto, União Liberal Republicana (ULR), 1926-1930. Um partido político na transição da I República para o Salazarismo, in «Polis», n. 4/5 1995, pp. I31-260; L. Farinha, Cunba Leal, Deputado e Ministro da República, cit. 
Il Prp, dal I8 dicembre 1923 fino alla fine della Prima repubblica, tornò ad essere a capo di tutti e 7 gli esecutivi che si avvicendarono, costituendo quasi sempre dei governi di coalizione con il Grupo parlamentar de Acção republicana e con gli indipendenti. La caduta e la formazione di questi governi dipendevano spesso dalle contestazioni e dalla preminenza dell'ala di sinistra o moderata del Prp.

Dopo il fallimento dei molti tentativi di varare una riforma del sistema politico e dell'unificazione delle forze repubblicane conservatrici, il Prp continuò a capo di una repubblica ormai screditata. La sfiducia nel sistema elettorale e nei partiti politici, fece in modo che vasti settori economici e sociali non si sentissero sufficientemente rappresentati e difesi dal parlamento, dai partiti e dal governo e cercassero una rappresentanza e un esercizio diretto del potere senza nessun tipo di mediazione politica.

Il discredito dei partiti come organizzazioni di mediazione della società con il potere politico e la necessità di creare un governo forte, al di fuori dei partiti, riscosse il favore anche delle organizzazioni padronali, dell'esercito e delle forze illiberali. Queste formazioni s'indirizzarono verso una strategia corporativa di rinforzo dell'organico, contro le istituzioni repubblicane e "la dittatura del Prp". Nel I924, dopo l'aumento di alcune imposte, le élite economiche e sociali entrarono in rotta di collisione con i governi capeggiati dal Prp. Le organizzazioni padronali decisero di formare un partito, l'União dos interesses económicos con l'obiettivo di intervenire attivamente in politica, dato che non si identificavano in nessuno dei partiti politici esistenti ed erano intimoriti dalle agitazioni sociali, dalla situazione economica del Paese, dall'avanzata del comunismo e dalla passività e inefficacia del potere politico. Anche la tensione tra l'esercito e i governi repubblicani continuò a crescere in questo periodo; nonostante i militari fossero profondamente divisi in termini politici e partitici, si verificò una crescente unità nell'intervento dei militari nella politica attraverso delle insurrezioni volte a risolvere i problemi corporativi delle forze armate e a creare un governo extra partitico che mettesse fine all'oligarchia del Prp. Anche l'estrema destra, divisa tra organizzazioni monarchiche e repubblicane, intraprese una strategia unitaria contro la "dittatura del Prp".

Un gruppo di intellettuali repubblicani liberali riuniti intorno alla rivista «Seara Nova» si rese conto del pericolo che correva la repubblica a causa dell'avanzata delle ideologie antiliberali in Portogallo, sia nella destra radicale ${ }^{22}$ (Integralismo lusitano, Cruzada nacional D. Nuno Àlvares Pereira e fascismo) sia nella estrema sinistra ${ }^{23}$ (comunismo e anarchismo), e quindi si impegnarono nella critica e nella riforma delle istituzioni repubblicane per conciliare l'eredità liberale con il rafforzamento

22. E. Castro Leal, Nação e Nacionalismo. A Cruzada D. Nuno Álvares Pereira e as Origens do Estado Novo, (19I8-1938), Lisboa, Edições Cosmos, 1999; A. Costa Pinto, Fascismo e Nazionalsindacalismo in Portogallo: 1914-1945, Roma, Antonio Pellicani, 200I.

23. C. Cunha, The Portuguese Communist Party's strategy for power, I92I-I986, New York, Garland Publishing, I992; J. Freire, Anarquistas e operários. Ideologia, ofício e práticas sociais: anarquismo e o operariado em Portugal 1900-1940, Porto, Afrontamento, 1992; J. Pacheco Pereira, Álvaro Cunhal: Uma biografia política, vol. I, Lisboa, Temas e Debates, I999. 
di un esecutivo forte. Il gruppo di «Seara Nova» attaccò la partitocrazia e caldeggiò la formazione di un governo nazionale "tecnico" con poteri straordinari conferiti dal parlamento come soluzione transitoria per poter riformare il sistema politico e rafforzare il liberalismo. Alcuni di questi intellettuali fecero parte brevemente del governo di Àlvaro de Castro, ma finirono per uscirne disillusi poiché non erano riusciti a riformare il sistema politico ${ }^{24}$.

Il Prp nonostante avesse conquistato nuovamente la maggioranza assoluta alle elezioni legislative del 8 novembre $\mathbf{1 9 2 5}$, facendo ricorso alla violenza, alla frode e agli accordi illeciti con i vari caciques, rimase profondamente isolato e ostracizzato dai partiti, dai sindacati, dalle associazioni padronali e militari. Queste organizzazioni passarono ad agire in modo sleale e semi illegale poiché avevano perso ogni fiducia nei confronti del sistema elettorale e politico. Come in altri paesi europei del primo dopoguerra, non ci fu un consenso di base sul funzionamento degli organi politici e la mancanza di legittimità democratica del regime impedì di superare la crisi delle istituzioni politiche repubblicane ${ }^{25}$.

L'ultimo governo della Prima repubblica, presieduto da António Maria da Silva (I7 dicembre 1925-30 maggio 1926) vide tutte le organizzazioni politiche di opposizione coinvolte in scandali economico-finanziari, in cospirazioni contro il governo del Prp e protagoniste di un profondo ostruzionismo nel parlamento, non consentì la votazione di alcune leggi. A questo scopo, utilizzarono vari stratagemmi, come l'abbandono delle sessioni per non raggiungere il quorum, l'aggressione di un deputato o, semplicemente, l'eccessiva durata dei discorsi parlamentari. La preparazione e l'esecuzione del golpe militare del 28 di maggio del 1926 vide la partecipazione di elementi di quasi tutti i partiti, sia di sinistra sia di destra. Tutti miravano a instaurare una dittatura transitoria che avrebbe permesso di chiudere con l'egemonia del Prp e di fondare le basi di un nuovo regime; tuttavia era solo questo il legame dell'unione delle forze politiche e militari che dettero luogo al movimento rivoluzionario che pose fine alla Prima repubblica ${ }^{26}$.

24. M. Villaverde Cabral, The «Seara Nova» Group (I92I-1926) and the ambiguities of Portuguese Liberal Elitism, in «Portuguese Studies», vol. 4, 1988, pp. I8I-195.

25. Cfr. The Breakdown of Democratic Regimes, a cura di J.J. Linz e A. Stepan Baltimore, Johns Hopkins U.P., 1978; J.J. Linz, La crisis de las democracias, in Europa en crisis 1919-1939, a cura di M. Cabrera, S. Juliá, P. Martín Aceña, Madrid, Editorial Pablo Iglesias, I99I, pp. 23I-28o; A. Costa Pinto, Portugal: Crises and early authoritarian takeover, in Conditions of democracy in Europe, I9I9-1939, a cura di D. Berg-Schosser, J. Mitchell, London, Macmillan Press, 2000, pp 354-380.

26. Cfr. R. Ramos, A Segunda Fundação (I890-1926), pp. 522-572; L. Farinha, O Reviralho: Revoltas republicanas contra a Ditadura e o Estado Novo 1926-1940, Lisboa, Editorial Estampa, I998; R. Ramos, O fim da República, in «Análise Social», vol. XXXIV, n. I53, 2000, pp. I059-I082; A. Afonso, História de uma conspiração: Sinel de Cordes e o 28 de Maio, Lisboa, Editorial Notícias, $200 I$. 


\section{Una transizione mancata verso la democrazia e i partiti di massa}

Durante tutto il periodo della Prima repubblica portoghese si mantenne un sistema multipartitico con un partito dominante e con una elevata instabilità politica. Il Portogallo ebbe 45 governi e 29 tentativi di golpe tra il ı9ıo e il ı926, presentando il maggior indice di instabilità governativa d'Europa nel periodo antecedente alla grande depressione, con una durata media per ogni governo di in7 giorni (I9I81926). Dietro allo stato iberico si posizionavano la Jugoslavia con una durata media di 154 giorni e la Spagna con $166^{27}$.

L'instabilità governativa derivava essenzialmente da due problemi. In primo luogo il Prp, pur dominando il parlamento, non era riuscito a creare un consenso interno che desse stabilità all'esecutivo. Questo varò, essenzialmente, governi di coalizione con l'appoggio degli altri partiti e dei deputati indipendenti, cosa che favorì il dissenso dentro il Prp e la nascita di fazioni. È per questo che molti governi caddero grazie ai voti dei parlamentari dello stesso Prp. In secondo luogo, ci fu una incapacità di trovare meccanismi pacifici di alternanza che dessero vita ad un governo stabile. Durante la Prima repubblica si continuò a vivere in un regime di «elezioni svolte ma alterate» ${ }^{28}$. Per questo, i partiti dell'opposizione non avendo fiducia nel funzionamento del sistema elettorale, ricorsero alla violenza e alle rivolte per arrivare al potere: «sono infatti le rivoluzioni e non le elezioni che assicurano l'alternanza politica ${ }^{29}$. Nella monarchia costituzionale il re poteva destituire il governo, nominare un nuovo esecutivo di opposizione, sciogliere il parlamento e indire nuove elezioni che normalmente erano vinte dal partito di governo. Questo meccanismo di alternanza politica, dopo un certo periodo, aveva permesso di smorzare le tensioni politiche. Durante la Prima repubblica il sistema costituzionale limitò questa pratica; e così molti politici ricorsero al colpo di Stato per arrivare al potere, visto che il sistema elettorale e costituzionale non gli dava questa possibilità. La violenza e la rivoluzione divennero fonti di legittimazione della sovranità del popolo e dell'alternanza politica.

Le varie forme di governo che furono sperimentate (governo partitico, di coalizione, di corrente, di nomina presidenziale) non ebbero un successo duraturo principalmente a causa delle lotte interne nel partito dominante nella Prima repubblica. Il Portogallo manteneva una instabilità politica endemica, anche se aveva apparentemente un sistema partitico capace di creare condizioni di grande stabilità politica. I partiti di estrema destra e sinistra erano minoritari (il Partido comunista português e i movimenti e partiti fascisti non riuscirono mai a eleggere né

27. J.J. Linz, La crisis de las democracias, in Europa en crisis 1919-1939, cit., p. 264.

28. A. Freire, Eleições, sistemas eleitorais e democratização: o caso português em perspectiva histórica e comparativa, in Eleições e Sistemas Eleitorais no Século XX Português, a cura di A. Freire, Lisboa, Edições Colibri, 20II, pp. 25-8I.

29. F. Marques da Costa, Bernardino Machado, in Os Presidentes da República Portuguesa, a cura di C. Pinto, Amadora, Círculo de Leitores, 200I, p. 69. 
un deputato né un senatore) e il Prp dominava il parlamento. La frammentazione partitica era presente soprattutto nei partiti del centro e non derivava da grandi divergenze ideologiche, ma da differenze personali tra $i$ vari leaders ${ }^{30}$.

In Portogallo il dopoguerra non portò riforme volte ad allargare la cittadinanza e non furono compiuti passi in avanti per introdurre il sistema proporzionale, o circoscrizioni elettorali più coerenti, come era avvenuto in Italia nel biennio 19I8-19. Fu mantenuto il sistema elettorale maggioritario con lista incompleta (ad esempio se la circoscrizione elettorale permetteva l'elezione di tre deputati, l'elettore poteva votarne solo due) che assicurava la rappresentanza dei principali partiti minoritari, ma dava, di fatto, vantaggio al partito dominante. Rimanevano alti tassi di astensione e i partiti politici continuavano a trascurare l'integrazione sociale e politica delle masse.

Il clientelismo nella Prima repubblica seguì il modello esistente nell'Europa del sud e mantenne alcune caratteristiche del periodo della monarchia ${ }^{31}$, acquisì solo un carattere più urbano e amministrativo. Il clientelismo si basava essenzialmente sul ruolo dei caciques e non su quello dei partiti politici intesi come organizzazioni. La rappresentanza degli interessi non era canalizzata dalla "organizzazione partito", ma da un insieme di notabili che curavano i propri interessi e quelli dei loro protetti, creando innumerevoli reti di favori incrociati, molte volte incompatibili all'interno dello stesso partito. L'organizzazione partitica si modernizzò poco, continuando con la stessa struttura personalistica di carattere oligarchico e basata sui caciques, il che allontanava la maggior parte dei membri dalle decisioni e teneva lontano la popolazione dalla politica. Le elezioni interne ai partiti e quelle politicoamministrative rimasero poco trasparenti e pertanto non si poterono consolidare nuovi partiti di integrazione sociale capaci di rinnovare i leaders e i procedimenti politici. La lotta per il potere continuò a basarsi sul controllo dell'esecutivo, il che rallentò il processo democratico, dato che si ebbe una minore integrazione degli interessi sociali e della partecipazione dei cittadini, anche a causa delle consuete frodi amministrative e della corruzione nei processi elettorali. Questa immagine

30. K. C. Schwartzman, The Social Origins of Democratic Collapse: The First Portuguese Republic in the Global Economy, Lawrence, Kansas, 1989.

31. Cfr. A. Lyttelton, El patronazgo de la Italia de Giolitti, in «Revista de Occidente», Tomo 43, n. I27, I973, Madrid, pp. 94-II7; J. Varela Ortega, Los Amigos Políticos. Partidos, Elecciones y Caciquismo en la Restauración (I875-1900), Madrid, Alianza Universidad, I977; L. Graziano, Clientelismo e sistema político. Il caso dell'Italia, Milano, FrancoAngeli, 1980; J. Cazorla Pérez, Del Clientelismo tradicional al clientelismo de partido: evolución y características, in «Working Papers», Institut de Ciènces Politiques i Socials, n. 55, 1992; Patronos y Clientes, a cura di E. Gellner et alii, Barcelona, Jucar Universidad, 1986; J. Moreno Luzon, Teoría del clientelismo y estudio de la política caciquil, in «Revista de Estudios Políticos», Madrid, n. 89, I995, pp. I9I-224; Id., El clientelismo político: Historia de un concepto multidisciplinar, in «Revista de Estudios Políticos», n. I05, 1999, pp. 73-95; Id., A historiografia sobre o caciquismo espanhol: balanço e novas perspectivas, in «Análise Social», n. I78, ICS, 2006, pp. 9-29. Política en penumbra. Patronazgo y clientelismo políticos en la España contemporánea, a cura di A. Robles Egea, Madrid, Siglo Veintiuno Editores, 1996; Democracia, elecciones y modernización en Europa, Siglos XIX y XX, a cura di S. Forner, Madrid, Cátedra, 1997; El poder de la influencia. Geografía del caciquismo en España (I875-1923), a cura di J. Varela Ortega, Madrid, Marcial Pons, 2001. 
dell'organizzazione clientelare giungeva all'opinione pubblica attraverso una campagna stampa sempre più aspra in relazione all'inefficacia dei partiti e del parlamento accusato di non essere riuscito ad approvare il bilancio la maggior parte degli anni. La modernizzazione e la democratizzazione non furono richieste in modo efficace dalla società, né furono promosse dai partiti. Questi dimostrarono di non essere preparati e motivati per raccogliere, modellare, e canalizzare verso il sistema politico gli interessi e le esigenze politiche di tutti gli strati sociali. Il proseguimento di pratiche clientelari dei partiti di notabili, la poca chiarezza nelle elezioni e l'inefficacia degli organi di governo accentuarono la crisi di legittimità delle istituzioni e spinsero fuori dal sistema politico ampie aree della società che svilupparono richieste massimaliste e attività rivoluzionarie ${ }^{32}$.

All'inizio degli anni venti i principali partiti della Prima repubblica non erano più "puri partiti di notabili", così come stava accadendo nello stesso periodo in Grecia e in Spagna ${ }^{33}$, visto che avevano iniziato un processo di modernizzazione e di adattamento ai nuovi tempi. In questo periodo infatti, i partiti, ancora lontani dall'essere "partiti di massa", erano comunque abbastanza evoluti rispetto a quelli di notabili del XIX secolo. Mentre nell'Europa occidentale si stavano affermando i partiti di massa, in Portogallo questi erano ancora dominati dalla élite parlamentare, dagli ex ministri e dalla loro rete clientelare composta da notabili regionali e caiciques locali34.

I principali dirigenti delle forze politiche repubblicane appartenevano alle élite urbane. Analizzandone le professioni è subito chiara la preminenza dei funzionari pubblici; tra questi, in particolare, i militari, gli alti funzionari dell'amministrazione pubblica, magistrati e professori. Altre categorie con un peso importante erano i giuristi, in particolare gli avvocati, e i medici. Rimanevano invece una parte minoritaria dei vertici dei principali partiti della Prima repubblica i commercianti, gli industriali e i proprietari terrieri. La classe dirigente aveva una elevata formazione accademica, tanto che più dell' $80 \%$ aveva compiuto studi di tipo universitario. In particolare le tre aree più rappresentate erano il diritto, l'alta formazione militare

32. Cfr. P. Tavares de Almeida, Eleições e Caciquismo no Portugal Oitocentista (I868-189o), Lisboa, Difer, I99I; F. Farelo Lopes, Poder Político e Caciquismo na I. ${ }^{a}$ República Portuguesa, cit.; Id., Os Partidos Políticos. Modelos e Realidades na Europa Ocidental e em Portugal, Oeiras, Celta Editora, 2004, pp. 29-49; Id., Direito de voto, regime de escrutínio e "eleições feitas" na I República Portuguesa e P. Tavares de Almeida, O sistema eleitoral e as eleições em Portugal (I895-I9Io): Uma perspectiva comparada e entrambi in Eleições e Sistemas Eleitorais no Século XX Português, a cura di A. Freire, Lisboa, Edições Colibri, 2oII, pp. 85-99 e IoI-I23; J. Manuel Gonçalves da Silva, O Clientelismo partidário durante a I República, cit.

33. Cfr. G. T. Mavrogordatos, Stillborn Republic. Social Coalitions and Party Strategies in Greece, 1922-1936, Berkeley, University of California Press, I983.

34. Cfr. S. Bernstein, Histoire du Parti Radical. La Recherche de L'Age D'Or (Igrg-1926), Paris, Presses de la Fondation National des Sciences Politiques, 1980; Il partito politico dalla Grande Guerra al fascismo. Crisi della rappresentanza e riforma dello Stato nell'età dei sistemi politici di massa (I9I8-I925), a cura di F. Grassi Orsini e G. Quagliarello, Bologna, il Mulino, 1996; G. M. Luebbert, Liberalismo, Fascismo o Socialdemocracia. Clases Sociales y orígenes políticos de los regímenes de la Europa de entreguerras, Zaragoza, Prensas Universitarias de Zaragoza, 1997; F. Farelo Lopes, Os Partidos Políticos, cit. 
e la medicina. Comparati con i partiti europei dell'epoca, in particolare con quelli dell'Europa del nord, l'élite partitica portoghese aveva una formazione accademica più elevata. Questa differenza era ancora maggiore se si comparano i dirigenti dei partiti portoghesi con quelli dei partiti di massa che si stavano affermando in Europa negli anni venti. Un'altra differenza era il maggior peso della qualifica militare nella élite partitica portoghese; inoltre, l'ascesa dei politici nella gerarchia del partito era più rapida e non era necessaria una esperienza nella politica locale per raggiungere ruoli di comando, come accadeva in molti partiti europei, pertanto era facile entrare nella direzione centrale di un partito prima dei 40 anni35.

Gli organi dei partiti portoghesi in cui erano prese le decisioni erano la direzione e il gruppo parlamentare. Le sedi locali dei partiti erano controllate dai caciques che avevano un'ampia autonomia grazie alla quale potevano siglare alleanze in base agli interessi strategici e familiari, non tenendo conto delle teorie ideologiche. I partiti continuavano ad avere un funzionamento interno carente e irregolare. Normalmente erano riuniti in confederazioni di commissioni locali indipendenti, capeggiate da caciques, poco coese e flessibili, con scarsi legami con le altre strutture partitiche. Nella maggior parte delle località la mobilitazione politica avveniva solo in occasione delle elezioni. L'organizzazione e la burocrazia erano estremamente deboli e si riassumevano, il più delle volte, in relazioni personali, in commissioni politiche con attività limitata al periodo elettorale, in centri associativi-partitici nelle città, in alcuni giornali nazionali e regionali. Il sistema di affiliazione dei militanti era ancora molto approssimativo ed era gestito dalle strutture locali, senza il controllo di quelle nazionali. I partiti avevano un apparato organizzativo abbastanza semplice e non esisteva al loro interno il ruolo del funzionario tipico delle forze politiche di massa. Il finanziamento era già composto parzialmente dai contributi individuali che gli iscritti pagavano in base al loro status, ma restava una parte che ancora dipendeva dalle entrate straordinarie provenienti dal patrimonio personale dei notabili. I partiti, infine, erano poco dottrinari e molto pragmatici; non ambivano a mobilitare le grandi masse popolari, né a trasmettere una immagine e una identità forte e consistente. Gli affiliati avevano una disciplina e una fedeltà ideologica debole e la relazione con gli elettori non era basata su meccanismi di delega, ma

35. Cfr. M. Roque Azevedo, Inquérito sobre o Partido Republicano Evolucionista (I9I2-I9Ig), in «Nova História», n. 2, I984, pp. 74-I22; R. Leite Pinto, União Liberal Republicana (ULR), I926-I930. (Um partido político na transição da I República para o Salazarismo), in «Polis», Revista de Estudos Jurídicos-Políticos, n. 4/5, I995, pp. I3I-260; M. Garcia Salazar Gonçalves da Silva, O Partido Reconstituinte, cit.; Parlamentares e Ministros da $I^{a}{ }^{a}$ República, a cura di A. H. de Oliveira Marques, Porto, Edições Afrontamento/Assembleia da República, 2000; Parliamentary Representatives in Europe 1848-2000. Legislative Recruitment and Careers in Eleven European Countries, a cura di H. Best e M. Cotta, Oxford, Oxford University Press, 20oo; Who Governs Southern Europe? Regime Change and Ministerial Recruitment (I850-2000), a cura di P. Tavaresde Almeida, A. Costa Pinto, N. Bermeo, London, Frank Cass, 2003; M. Dias Santos, Os Monárquicos e a República Nova, Coimbra, Quarteto, 2003; A. B. Malheiro da Silva, Sidónio e Sidonismo, cit.; P. Tavares de Almeida, P. Jorge Fernandes, M. Carvalho dos Santos, Os Deputados da I. ${ }^{a}$ República Portuguesa: Inquérito Prosopográfico, in «Revista de História das Ideias», 27, 2006, pp. 399-417; A. J. Sousa Monteiro de Queirós, A Esquerda Democrática e o final da Primeira República, cit.; E. Castro Leal, Partidos e Programas, cit. 
di fiducia. Il loro obiettivo centrale era ottenere un accesso privilegiato alle risorse statali e allettare il maggior numero di persone influenti a livello regionale e locale, dato che questi schieramenti politici erano composti da un insieme di notabili e dai loro sostenitori locali che arrecavano prestigio, capacità tecnica e capitali al partito. La coesione interna si manteneva attraverso la elargizione di benefici per la collettività (una strada o una scuola) e individuali (raccomandazioni per trovare lavoro o risoluzioni di problemi burocratici con l'amministrazione pubblica) che il notabile garantiva ai propri sostenitori in cambio di appoggio durante la campagna elettorale. L'indisciplina, la frammentazione e le scissioni in questo tipo di partiti erano frequenti, dovute principalmente alle divergenze personali e alle lotte di potere. Le scissioni davano luogo a una nuova associazione tra alcuni notabili e i loro affiliati; questa aveva nuovi leaders e nuove ramificazioni in tutto il paese e, a volte, rappresentava il primo passo per la formazione di un nuovo partito ${ }^{36}$.

Il Prp e le altre forze politiche portoghesi non si trasformarono in partiti di massa. Si continuò ad avere infatti una rottura e un allontanamento progressivo tra le rivendicazioni urbane, democratiche e partecipative e il mantenimento di una struttura elitista basata su una concezione monopolista, gerarchica e chiusa del potere. Tuttavia, è giusto notare come questa realtà non era né monolitica né perenne. Quasi tutti i partiti portoghesi iniziarono un avvicinamento verso un modello di partito moderno e funzionale, in particolare il Prp, seguendo il modello degli altri schieramenti politici di notabili europei, come il Partito radicale francese, il Maurismo e la Lliga regionalista catalana in Spagna. In Portogallo, tuttavia, continuarono a rimanere anche gruppi con una organizzazione tradizionale, con una base quasi esclusivamente parlamentare e con una debole o inesistente rete organizzativa a livello nazionale, come il Grupo parlamentar de acão repubblicana $(1924-25)^{37}$.

Visto che alcuni partiti di notabili già a partire dagli anni venti non avevano più le stesse caratteristiche dei partiti del XIX secolo, una certa storiografia spagnola rimodellò il concetto di "partito di notabili" di Max Weber e il concetto di "partito di quadri" di Maurice Duverger. Gli schieramenti politici che non si modernizzarono continuarono a essere designati come partiti di notabili, mentre quelli che iniziarono una modernizzazione furono definiti partiti di quadri. In questo modo i partiti di quadri rappresentarono una fase di transizione nel processo evolutivo: persero alcune delle caratteristiche tradizionali dei partiti di notabili, e si dotarono di una struttura burocratica e organizzativa quasi permanente, di una élite più diversificata e preparata per la politica di massa ottenendo una maggiore mobilitazione e inquadramento degli iscritti. Questi partiti, tuttavia, non avevano ancora le caratteristiche di quelli si massa; utilizzavano simultaneamente una pratica politica moderna nelle città e una di tipo clientelare nelle zone rurali. Tra

36. Cfr. F. Farelo Lopes, Poder Político e Caciquismo na I. ${ }^{a}$ República Portuguesa, cit.; J. M. Garcia Salazar Gonçalves da Silva, O Partido Reconstituinte, cit.; A. José Sousa Monteiro de Queirós, A Esquerda Democrática e o final da Primeira República, cit.; E. Castro Leal, Partidos e Programas, cit.

37. J. M. Garcia Salazar Gonçalves da Silva, O Partido Reconstituinte, cit. 
i mezzi moderni, ad esempio, le visite nella circoscrizione durante la campagna elettorale, gli incontri con i votanti, il comizio, l'elezione dei candidati all'interno del partito, l'elaborazione di un programma e la concessione dei benefici per tutta una comunità e non solo per alcuni individui. Le pratiche tradizionali, tuttavia, continuarono a persistere, in particolare nelle zone rurali, come la compravendita del voto, l'azione dei caciques, la violenza, la coercizione dei lavoratori da parte del padrone, l'ingerenza dei prefetti e degli altri membri della amministrazione pubblica, la frode e la manipolazione finale dei risultati elettorali ${ }^{8}$.

I gruppi che avevano una struttura simile ai partiti di quadri si trovavano soprattutto nelle città, in particolare a Lisbona, mentre nelle aree rurali continuavano a mantenere una struttura e una pratica politica tipica dei partiti di notabili. Rispetto ad altri paesi del sud Europa ci fu un notevole ritardo nella modernizzazione delle forze politiche. I partiti di quadri spagnoli anche se continuarono con le pratiche clientelari, dettero segno di seguire le correnti democratiche dell'Europa del dopoguerra, con un programma riformista graduale che controbilanciava le minacce rivoluzionarie ${ }^{39}$. I partiti di quadri italiani incontrarono molte difficoltà ad adattarsi all'allargamento del suffragio e al sistema proporzionale introdotto nel i9i9 che aveva permesso il rafforzamento dell'influenza politica dei moderni partiti di integrazione sociale, come quello socialista o popolare ${ }^{40}$. Il ritardo nella modernizzazione dell'economia e della società portoghese e il carattere rivoluzionario del regime repubblicano ritardarono la metamorfosi interna dei partiti portoghesi analogamente a quanto stava accadendo in alcuni paesi europei - una cooperazione con un lento processo di democratizzazione - anche se questo avrebbe significato, in un medio periodo, il superamento di queste forze politiche di notabili da parte

38. Cfr. L. Arranz Notario, Modelos de partido, in «Ayer», n. 20, 1995, pp. 8I-IIo; M. Sierra, La política del pacto. El sistema de la restauración a través del partido conservador sevillano (I874-1923), Sevilla, Diputación de Sevilla, 1996; M. A. Peña Guerrero, Clientelismo político y poderes periféricos durante la Restauración. Huelva I874-I923, Huelva, Universidad de Huelva, I998; Elpoder de la influencia, a cura di J. Varela Ortega, cit.; J. Moreno Luzón, A historiografia sobre o caciquismo espanhol, cit., pp. 9-29.

${ }_{39}$ Cfr. M. Suárez Cortina, El Reformismo en España. Republicanos y Reformistas bajo la Monarquía de Alfonso XIII, Madrid, Siglo Veintiuno, 1986; Elpoder de la influencia, a cura di J. Varela Ortega, cit.

40. Cfr. M. Ridolfi, Il PSI e la nascita del partito di massa, I892-1922, Roma, Laterza, 1992; S. Noiret, La nascita del sistema dei partiti nell'Italia contemporanea. La proporzionale del I9I9, Roma, Piero Lacaita editore, 1994; Id., La introducción del sufragio universal y de la representación proporcional en Italia en I9I8-19I9: una frágil modernización democrática, in Democracia, elecciones y modernización en Europa, Siglos XIX y XX, a cura di S. Forner, Madrid, Cátedra, 1997, pp. 73-95; P. Pombeni, Partiti e sistemi politici nella storia contemporanea (I830-I968), Bologna, il Mulino, I994, pp. 485-505; Il partito politico dalla Grande Guerra al fascismo, cit.; S. Noiret, Le campagne elettorali dell'Italia liberale: dai comitati ai partiti, in, Idee di rappresentanza e sistemi elettorali in Italia tra Otto e Novecento, a cura di P. L. Ballini, Venezia, Istituto Veneto di Scienze Lettere ed Arti, 1997, pp. 383-454; M. Ridolfi, Interessi e passioni. Storia dei partiti politici italiani tra l'Europa e il Mediterraneo, Milano, Bruno Mondadori, I999; M. Tarchi, Italy: Early Crisis and Fascist Takeover, in Conditions of Democracy in Europe, I9I939. Systematic Case Studis, a cura di D. Berg-Schlosser, J. Mitchell, London, Macmillan Press, 2ooo, pp. 294-320; L. Lotti, I Partiti dal sistema uninominale alla proporzionale: la classe dirigente liberale di fronte all'affermazione dei movimenti di massa, in España e Italia en la Europa Contemporánea: desde finales del siglo XIX a las Dictaduras, a cura di F. García Sanz, Madrid, Consejo Superior de Investigaciones Científicas, 2002, pp. 69-76. 
dei partiti di massa. La transizione verso la democrazia in Portogallo non dipese solo dalla volontà delle élite politiche nazionali e locali, ma principalmente dalla società stessa, che non era ancora pronta negli anni venti, né a sostenere un sistema di democrazia rappresentativa né un tipo di partito, come quello socialista comunista o fascista, volti a integrare le masse al loro interno. I partiti portoghesi inseriti in una società fragile continuarono a trovare la loro base nelle élite, rifuggendo le masse. Il sistema politico e sociale portoghese aveva alcune caratteristiche che lo avvicinavano più ai regimi politici del sud America e dell'est Europa che non a quello dell'Europa occidentale, con una società rurale, una industrializzazione tardiva e una economia arcaica, inserita in un sistema politico con una tradizione parlamentare ma di rivalità e pluralismo limitato, con un basso livello di mobilitazione politica; elementi che facilitarono la transizione verso una dittatura con caratteristiche tradizionali, dove la mobilitazione fascista non era necessaria o proprio pregiudiziale per proteggere gli interessi nelle élite dominanti ${ }^{4+}$.

\section{traduzione dal portoghese di Annarita Gori}

4r. Cfr. N. P. Mouzelis, Politics in the Semi-Periphery. Early Parliamentarism and Late Industrialization in the Balkans and Latin America, London, Macmillan, I986; M. Dogan, Romania, I919-1938, in Competitive elections in developing countries, a cura di Myron Weiner; Ergun Ozbudun, Durham, Duke U.P., 1987, pp. 369-389; F. Farelo Lopes, Poder Político e Caciquismo na I. ${ }^{a}$ República Portuguesa, cit.; Id., Os Partidos Políticos, cit.; J. M. Gonçalves da Silva, O Clientelismo partidário durante a I República, cit.; R. Ramos, A Segunda Fundação, cit.; Id., Foi a Primeira República um regime liberal?, cit.; A. Costa Pinto, Portugal: Crises and early authoritarian takeover, cit. 